\title{
Slow dynamics of Zero Range Process in the Framework of Traps Model
}

\author{
Kai Qi, ${ }^{1}$ Ming Tang,, , A Aixiang Cui, ${ }^{1}$ and Yan $\mathrm{Fu}^{1}$ \\ ${ }^{1}$ Web Sciences Center, University of Electronic Science and Technology of China, Chengdu 610054, People's Republic China
}

(Dated: November 7, 2018)

\begin{abstract}
The relaxation dynamics of zero range process (ZRP) has always been an interesting problem. In this study, we set up the relationship between ZRP and traps model, and investigate the slow dynamics of ZRP in the framework of traps model. Through statistical quantities such as the average rest time, the particle distribution, the two-time correlation function and the average escape time, we find that the particle interaction, especially the resulted condensation, can significantly influence the dynamics. In the stationary state, both the average rest time and the average escape time caused by the attraction among particles are obtained analytically. In the transient state, a hierarchical nature of the aging dynamics is revealed by both simulations and scaling analysis. Moreover, by comparing the particle diffusion in both the transient state and the stationary state, we find that the closer ZRP systems approach the stationary state, the more slowly particles diffuse.
\end{abstract}

PACS numbers: 05.40.Fb,05.60Cd,89.75.Hc

The investigation of diffusion process plays a very important role in exploring the structures of systems and uncovering the physical mechanisms of dynamics in particle systems [14]. Recently, with the booming research results in the field of complex networks, particle diffusion in complex networks has once again become a hot problem [5-20]. For example, Noh et al. studied random walks in scale-free (SF) networks and found that the ratio between a coordination number and a characteristic relaxation time for each node essentially determines the MFPT [11].

A common feature of above studies is that there is no interaction among particles at each node. Actually, particle interaction is unavoidable and can be found in many real networks where it plays an important role in the dynamical processes in networks. One simple way to introduce the interactions among stochastic particles is so-called zero range process (ZRP), which has been recently adopted to investigate particle condensation in complex networks [21-25]. It is shown that this condensation transition appears in a number of unexpected contexts such as wealth condensation in macroeconomies [26], jamming in traffic [27-30], coalescence in granular systems [31, 32], and gelation in networks [33, 34]. To understand the influence of ZRP interaction on the particle diffusion, we made an important first step to study another aspect of ZRP in scale-free networks, i.e., the diffusion features [35]. We found that the statistical quantities of diffusion can be significantly reduced by the condensation and can be figured out by the rest time of a particle staying at a node. In addition to these statistical quantities in the stationary state, however, the relaxation dynamics of ZRP are fully overlooked.

Recently, a traps model with interaction, in which particles are attracted at each node (i.e. local minima) with the potential-energy landscape [36], was put forward to study the connection between the network of the potential-energy landscape and the glassy dynamics [37]. At low temperatures, the relation between the energy and the degree of a minimum can

*Electronic address: tangminghuang521@ @otmail.com result in the slow dynamics of glassy systems. This provided a systematic integration of tools and concepts to investigate how network structures impact the particle diffusion. In this letter, we will try to set up the relationship between ZRP and traps model, and then investigate the slow dynamics in the ZRP interaction in the framework of traps model.

Firstly, we briefly introduce the ZRP model. In complex networks, ZRP interaction means that a particle interacts only with other particles staying at the same node. Suppose $N$ particles are randomly put in a network with $L$ nodes and each node $i$ can be occupied by any integer number of particles $n_{i}=0,1,2, \cdots, N$. Due to interaction, usually only part of the particles at a node can jump out and hop into its neighboring nodes, which makes the particles be redistributed among all nodes. We divide the diffusion process of particles into two steps. In the first step, some particles at node $i$ will jump out because of the interaction among the particles. Suppose a particle at node $i$ jumps out at the rate $p\left(n_{i}\right)=n_{i}^{\delta}$ with $\delta \in[0,1]$ [21-23], where the parameter $\delta$ can be used to characterize the nature of interaction among particles at the node. In the second step, each jumping particle hops from the node $i$ to one of its neighbors $j$ randomly.

In the stationary state, there is a condensation threshold $\delta_{c}=1 /(\gamma-1)$, and a finite fraction of the total particles will be condensed to the hubs for $\delta<\delta_{c}[21-23]$. Generally, the particle density $\rho=N / L$. In the mean field approach, the description of $n_{i}$ for each node is replaced with the mean occupation number $m_{k}$ for the nodes with the same degree $k$, i.e., $m_{k}$ is the average of all the $n_{i}$ at the nodes with degree $k$. Hence $m_{k}$ is not necessarily an integer. In this framework, it is shown that the number of mean occupation particle in the stationary state is [23]

$$
\begin{aligned}
& m_{k}=k / k_{c}, k<k_{c} \\
& m_{k}=\left(k / k_{c}\right)^{1 / \delta}, k \geq k_{c}
\end{aligned}
$$

where the crossover degree $k_{c}$ denotes the degree for $m_{k}=1$, and is given by [35]

$$
k_{c} \simeq\left\{\begin{array}{l}
{\left[\frac{A}{\rho(-\gamma+1+1 / \delta)}\right]^{\delta} k_{\max }^{1-\delta / \delta_{c}}, \quad \text { for } \delta<\delta_{c}} \\
{\left[\frac{A}{\rho}\right]^{\delta}\left[\ln k_{\max }\right]^{\delta_{c}}, \quad \text { for } \quad \delta=\delta_{c} .}
\end{array}\right.
$$


For $\delta>\delta_{c}$, there is no condensation, and the crossover degree $k_{c}$ is given by

$$
\rho \simeq \frac{A k_{c}^{-1}}{\gamma-2}\left(k_{0}^{-\gamma+2}-k_{c}^{-\gamma+2}\right)+\frac{A k_{c}^{-\gamma+1}}{\gamma-1-1 / \delta} .
$$

To gain further insight into the relaxation dynamics of the ZRP systems [35], we first set up the relationship between ZRP and traps model. Treating each node as a trap with temperature $T$ and depth $E_{k}$, we obtain a trapping network where both $T$ and $E_{k}$ can be determined by the jumping rate $p(m)$. As pointed out in our previous work [35], the jumping rate has different expressions

$$
p\left(m_{k}\right)= \begin{cases}m_{k}=k / k_{c}, & \text { for } \quad k<k_{c} \\ m_{k}^{\delta}=k / k_{c}, & \text { for } \quad k \geq k_{c}\end{cases}
$$

That is, $p\left(m_{k}\right)$ is proportional to the degree $k$ and has the same expression for all degree $k$. As the jumping rate $p(m)$ represents the number of particles jumping out of a given node per unit time, the rest time $\tau_{k}$ is $m_{k} / p\left(m_{k}\right)$, which is given by

$$
\tau_{k}=\left\{\begin{array}{l}
1, \quad \text { for } \quad k<k_{c} \\
{\left[\frac{k}{k_{c}}\right]^{1 / \delta-1}, \quad \text { for } \quad k \geq k_{c} .}
\end{array}\right.
$$

Obviously, larger $p(m)$ corresponds to higher $T$ and smaller $E_{k}$, and vice versa. In this way, we compare the rest time $\tau_{k}=m_{k} / p\left(m_{k}\right)$ in ZRP with the trapping time $\tau_{k}=e^{\beta E_{k}}$ in trap model where $\beta=1 / T$ [37]. It is easy to obtain: For $k<$ $k_{c}, \beta E_{k}=0$; for $k \geq k_{c}, \beta E_{k}=\frac{1-\delta}{\delta} \log \left(\frac{k}{k_{c}}\right)$. Letting $T=$ $\frac{1}{\beta}=\delta /(1-\delta)$, then we have

$$
\begin{aligned}
& E_{k}=0, \quad \text { for } \quad k<k_{c} ; \\
& E_{k}=\log (k)-\log \left(k_{c}\right), \quad \text { for } \quad k \geq k_{c} .
\end{aligned}
$$

We see that $\delta \rightarrow 0$ corresponds to $T \rightarrow 0$, the condensation threshold $\delta_{c}$ corresponds to $T_{c}=1 /(\gamma-2)$, and $\delta \rightarrow 1$ corresponds to $T \rightarrow \infty$. From Eq. (6) we see that larger $k$ corresponds to larger $E_{k}$, i. e., deeper trap. In Ref. [37], the average rest time $\tau(\delta)$ is exactly the average rest time $\langle\tau\rangle=\langle k\rangle^{-1} \sum_{k} k P(k) \tau_{k}$, indicating that the framework of ZRP is equivalent to that of the trap model. In the thermodynamic limit of $L, N \rightarrow \infty$, the average rest time before a hop is $\tau(\delta)=\int_{k_{0}}^{k_{\max }} \frac{k P(k) \tau_{k}}{\langle k\rangle} d k$. Substituting Eq. (5) into this equation, we have

$$
\tau(\delta)=\frac{k_{c}}{\langle k\rangle} \int_{k_{0}}^{k_{\max }} m_{k} P(k) d k=\frac{\rho k_{c}}{\langle k\rangle},
$$

which depends on $\delta$ through $k_{c}$. For $\delta \leq \delta_{c}$, substituting Eq. (2) into Eq. (7) we obtain

$$
\tau(\delta) \simeq\left\{\begin{array}{l}
\frac{\rho}{\langle k\rangle}\left[\frac{A}{\rho(-\gamma+1+1 / \delta)}\right]^{\delta} k_{\max }^{1-\delta / \delta_{c}}, \quad \text { for } \delta<\delta_{c} \\
\frac{\rho}{\langle k\rangle}\left[\frac{A}{\rho}\right]^{\delta}\left[\ln k_{\max }\right]^{\delta_{c}}, \quad \text { for } \quad \delta=\delta_{c} .
\end{array}\right.
$$

For $\delta_{c}<\delta<1$, the crossover degree $k_{c}$ can be obtained from Eq. (3). Especially, for the case of $\delta=1$, we have $k_{c}=\langle k\rangle / \rho$

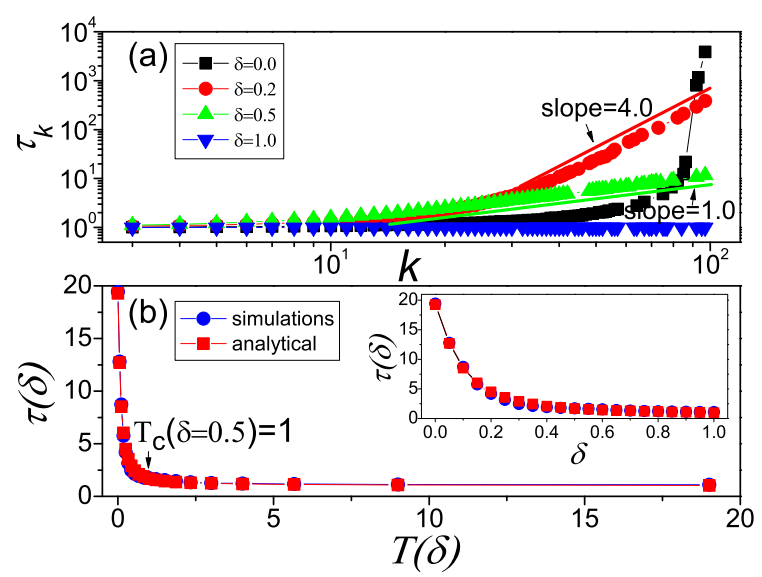

FIG. 1: (color online). (a) The rest time $\tau_{k}$ vs degree $k$ for $\delta=$ $0.0,0.2,0.5,1.0$. The solid lines represent the slopes $s=1 / \delta-1=$ 1.0 and 4.0 , respectively. (b) The average rest time $\tau(\delta)$ versus the temperature $T(\delta)$. The "squares" represent the numerical simulations and the "circles" represent the theoretical results according to Eq. (7). The inset shows how $\tau(\delta)$ changes with $\delta$.

when $m_{k_{c}}=1$ [23]. Substituting it into Eq. (7] we have $\tau=1$, which is consistent with the case of random walk.

To confirm the theoretical results, we make numerical simulations in an uncorrelated configuration model (UCM) [38]. We first construct an UCM network with size $L=10^{4}$ and degree distribution $P(k) \sim A k^{-3}$, where $A \approx 13$. Other parameters of this network are $\langle k\rangle \approx 5, k_{0}=3$, and $k_{\max }=97$, where $k_{0}$ and $k_{\max }$ denote the minimum and maximum degree of the network, respectively. We set the particle density $\rho=1$ and let the particles hop in the network. In the stationary state, we calculate the mean rest time $\tau_{k}$ at the nodes with the same degree $k$, and figure out their average $\tau$. The results shown in Fig. 1 1 are consistent with the theoretical predictions in Eqs. (5) and (7). As shown in Fig. 1 (b), the average rest time $\tau(\delta)$ decreases significantly with the increase of $\delta$ for $\delta<\delta_{c}$, indicating that the diffusion is remarkably slowed down in the condensation phase.

In the stationary state, a normalized particle distribution $P_{e q}(k)$, which is defined as the probability for a particle to be in any node with degree $k$, is given by $P_{e q}(k)=$ $P(k) m_{k} / \rho$, and thus

$$
P_{e q}(k) \sim\left\{\begin{array}{l}
\frac{A k^{-\gamma+1}}{\rho k_{c}}, \quad \text { for } k<k_{c} \\
\frac{A k^{-\gamma+1 / \delta}}{\rho k_{c}^{1 / \delta}}, \quad \text { for } k \geq k_{c} .
\end{array}\right.
$$

On the other hand, the relaxation dynamics in the condensation phase is an interesting problem [22, 23]. Here we investigate how $P\left(k, t_{w}\right)$ converges to $P_{e q}(k)$ in the transient state. Until now, there is no exact theory for the relaxation dynamics of the ZRP, and almost all the studies have been investigated by Monte Carlo simulations. In the transient period, the simulated results stir up one conjecture that the relaxation dynamics has a hierarchical nature [22, 37]: At first, subnetwork with small degree nodes is stable, and then larger degree 
regions progressively equilibrate. In fact, the nodes with small degrees correspond to shallow minima, which take less time to explore, while the nodes with large degrees are deep traps which take longer time to equilibrate. At time $t_{w}$, one can suppose that the nodes with $k \leq k_{w}$ are "at equilibrium", while the nodes with $k \gg k_{w}$ are still in the random walk regime. It turns out that the particle distribution behaves in each regime as

$$
P\left(k ; t_{w}\right) \sim \begin{cases}k^{-\gamma+1} & \text { for } \quad k \lesssim k_{v} \\ k^{-\gamma+1 / \delta} & \text { for } \quad k_{v} \lesssim k \lesssim k_{w} \\ k^{-\gamma+1} & \text { for } k_{w} \lesssim k .\end{cases}
$$

Like $k_{c}$ in the stationary state, $k_{v}$ plays the role of the crossover degree scale in a subnetwork with the largest degree $k_{w}$ and the network size $L^{\prime} \sim k_{w}^{\delta_{c}}$, that is,

$$
k_{v} \sim \begin{cases}k_{w}^{1-\delta / \delta_{c}} & \text { for } \delta<\delta_{c} \\ \left(\ln k_{w}\right)^{\delta_{c}} & \text { for } \delta=\delta_{c}\end{cases}
$$

Considering that the total time $t_{w}$ is the sum of the trapping times of the visited nodes, which is dominated by the longest one $\tau_{k}$ from Eq. (5), we obtain $k_{v} \sim t_{w}^{\left(\delta_{c}-\delta\right) /(1-\delta)}$ and $k_{w} \sim t_{w}^{\delta_{c} /(1-\delta)}$ for $\delta<\delta_{c}$ in the looped networks. Figure 2 (a) and (b) show indeed that the whole non-equilibrium distribution can be cast into the scaling form

$$
P\left(k ; t_{w}\right)=P(k) F\left(k / t_{w}^{\left(\delta_{c}-\delta\right) /(1-\delta)}\right),
$$

where $F$ is a scaling function such that $F(x)$ displays the scaling like Eq. (1) at small $x$, and $F(x) \sim x$ at large $x$. In addition, as shown in Fig.2 (c) and (d), the whole non-equilibrium distribution also displays the scaling

$$
P\left(k ; t_{w}\right)=t_{w}^{-\delta_{c} /(1-\delta)} G\left(k / t_{w}^{\delta_{c} /(1-\delta)}\right),
$$

where $G(x) \sim x^{1-\gamma}$ at large $x$.

This evolution takes place until the nodes with the largest degree $k_{\max }$, equilibrate. For an uncorrelated scale-free network, $k_{\max } \sim L^{1 / 2}$ so that the equilibration time is

$$
T_{e q} \sim k_{\max }^{(1-\delta) / \delta_{c}} \sim L^{1-\delta} .
$$

It is consistent with the results in Ref. [22, 23].

The evolution of $P\left(k ; t_{w}\right)$ in the condensation phase corresponds to the aging dynamics of the system, which has a hierarchical nature. This dynamics is also investigated through a two-time correlation function $C\left(t_{w}+t, t_{w}\right)$ between the states of the system at times $t_{w}$ and $t_{w}+t$, defined as the the average probability that a particle has not changed trap between $t_{w}$ and $t_{w}+t[36]$ : this amounts to considering that the correlation is 1 within one trap and 0 between distinct traps. The probability that a walker remains in trap $i$ longer than $t$ is simply given by $\exp \left(-t / \tau_{i}\right)$, so that

$$
C\left(t_{w}+t, t_{w}\right)=\int d k P\left(k ; t_{w}\right) e^{-t / \tau_{k}} .
$$
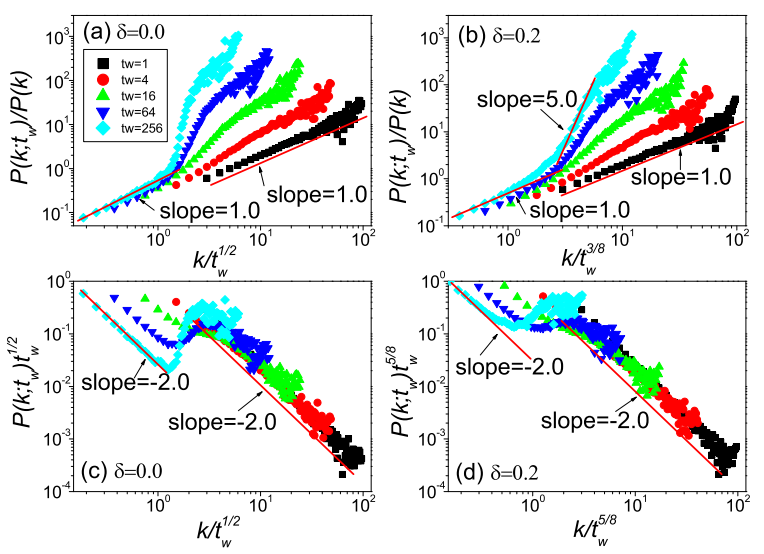

FIG. 2: (color online). The scaling form of non-equilibrium distribution with $\delta=0.0,0.2$ in the transient state. Data in both (a) and (b) obey the scaling in Eq. [12, those in both (c) and (d) obey the scaling in Eq. 13.

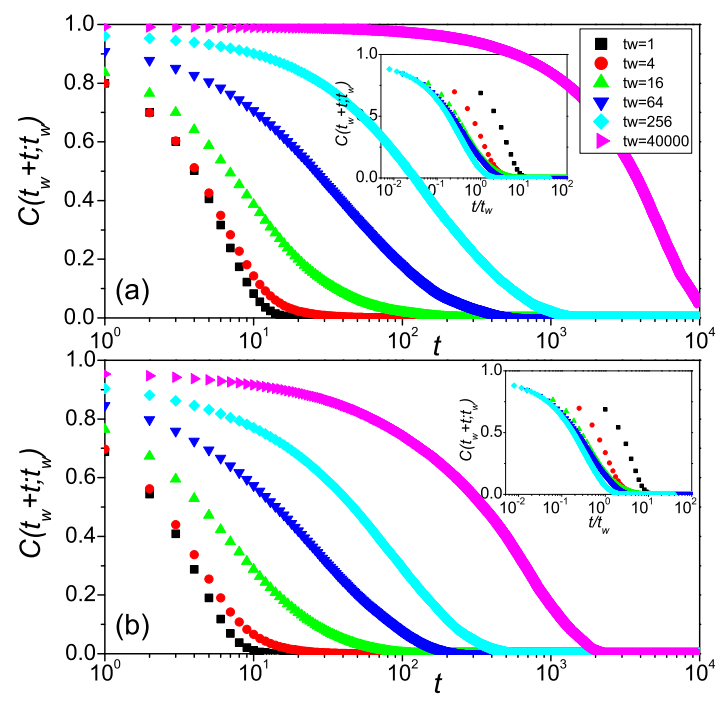

FIG. 3: (color online). $C\left(t_{w}+t, t_{w}\right)$ vs $t$ with $\delta=0.0$ (a) and $\delta=0.2(\mathrm{~b})$ in the condensation phase. The insets show $C\left(t_{w}+\right.$ $\left.t, t_{w}\right)$ vs $t / t_{w}$.

In Fig. 3, simulations show that the closer ZRP systems approach the stationary state, the more slowly particles diffuse, which seems like the critical slowing down in phase transition. As the stationary state is approached, more and more particles are trapped in hubs, and it's very difficult for these particles to escape from hubs. Thus, the correlation length (i. e., the characteristic time for the two-time correlation to disappear) becomes longer and longer [23]. For ZRP dynamics in uncorrelated scale-free networks, it is obvious that the correlation function doesn't obey the simple aging $C\left(t_{w}+t, t_{w}\right)=g\left(t / t_{w}\right)$ because of the increasingly particle attraction in the transient state.

Aging properties of the system can also be measured 

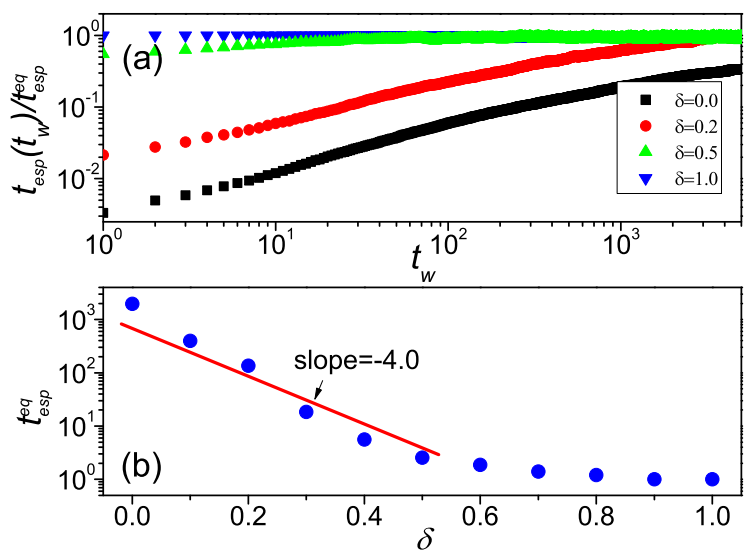

FIG. 4: (color online). The average escape time $t_{e s c}$ in both the transient state and the stationary state. (a) $t_{e s c}\left(t_{w}\right) / t_{e s c}^{e q}$ vs $t_{w}$ for $\delta=0.0,0.2,0.5,1.0$ in the transient state. (b) $t_{e s c}^{e q}$ vs $\delta$ where the solid line represents the slope $s=-\log _{10} L=-4.0$ from Eq. (16).

through the average escape time $t_{e s c}\left(t_{w}\right)$ required by the random walker to escape from the node it occupies at time $t_{w}$. We define $t_{e s c}=\left\langle t^{\prime}\right\rangle-t_{w}$, where $t^{\prime}>t_{w}$ is the time of the first jump performed by the walker after $t_{w}$, which gives $t_{e s c}\left(t_{w}\right)=\int d k \tau_{k} P\left(k ; t_{w}\right)$. For small $t_{w}$ with respect to the equilibration time, $t_{e s c}$ is growing due to the evolution of $P\left(k ; t_{w}\right)$. After a long time, $m_{k}\left(t_{w}\right) \rightarrow m_{k}^{e q}$ in any finite system, and then we have $t_{e s c}^{e q}=\int_{k_{0}}^{k_{\max }} d k \frac{P(k) m_{k} \tau_{k}}{\rho}$. Substituting Eq. (1) and (5) into this equation, we can numerically calculate $t_{e s c}^{e q}$ for the different $\delta, t_{e s c}^{e q}=\int_{k_{0}}^{k_{c}} \frac{P(k) m_{k}}{\rho} d k+$ $\int_{k_{c}}^{k_{\max }} \frac{P(k) m_{k}^{2-\delta}}{\rho} d k$. For $\delta \leq \delta_{c}$, the system is in the condensation phase. The nodes with $k_{c} \leq k \leq k_{\max }$ have the capacity to accommodate most particles. Therefore, we have $t_{\text {esc }}^{e q} \simeq \int_{k_{c}}^{k_{\max }} \frac{P(k) m_{k}^{2-\delta}}{\rho} d k$, and obtain the scaling as follows

$$
t_{e s c}^{e q} \sim k_{\max }^{(\gamma-1)(1-\delta)} \sim L^{1-\delta} .
$$

In summary, we have set up the relationship between ZRP and traps model, i. e., $T=\delta /(1-\delta)$, and then investigated the relaxation dynamics of ZRP in the framework of traps model. The particle interaction, especially the resulted condensation, is found to significantly influence the dynamics. In the stationary state, a rest time $\tau(\delta)=\rho k_{c} /\langle k\rangle$ is caused by the attraction among particles. In the transient state, a hierarchical nature of the aging dynamics for $\delta<\delta_{c}$ is revealed by means of the scaling analysis of particle distribution. In addition, the equilibration time has the scaling $T_{e q} \sim L^{1-\delta}$. Moreover, the slow dynamics in both the transient state and the stationary state have been compared by two important statistical measures, the two-time correlation function and the average escape time. Both simulations and scaling analysis show that the closer ZRP systems approach the stationary state, the more slowly particles diffuse. At a long time, the average escape time in the condensation phase displays the scaling $t_{e s c}^{e q} \sim L^{1-\delta}$. It is expected that the present work will be useful for understanding the slow dynamics of condensation in the real world.

\section{Acknowledgments}

This work is supported by the NNSF of China (Grants No. 11105025, 61103109), China Postdoctoral Science Foundation (Grant No. 20110491705), the Specialized Research Fund for the Doctoral Program of Higher Education (Grant No. 20110185120021), and the Fundamental Research Funds for the Central Universities (Grant No. ZYGX2011J056).
[1] Hughes B D 1996 Clarendon Press Oxford

[2] Redner S 2001 Cambridge University Press New York

[3] ben-Avraham D and Havlin S 2000 Cambridge University Press Cambridge

[4] Woess W 2000 Cambridge University Press Cambridge

[5] Adamic L A, Lukose R M, Puniyani A R and Huberman B A 2001 Phys. Rev. E 64046135

[6] Yang S 2005 Phys. Rev. E 71016107

[7] Sood V, Redner S and ben-Avraham D 2005 J. Phys. A: Math. Gen. 38109

[8] Almaas E, Kulkarni R V and Stroud D 2003 Phys. Rev. E 68 056105

[9] Jasch F and Blumen A 2001 Phys. Rev. E 64066104

[10] Bollt E M and Ben-Avraham D 2005 New J. Phys. 726

[11] Noh J D and Rieger H 2004 Phys. Rev. Lett. 92118701

[12] Gallos L K 2004 Phys. Rev. E 70046116

[13] Kittas A, Carmi S, Havlin S and Argyrakis P 2008 Europhys. Lett. 8440008

[14] Baronchelli A, Catanzaro M and Pastor-Satorras R 2008 Phys. Rev. E 78011114
[15] Juhász R 2008 Phys. Rev. E 78066106

[16] Baronchelli A and Loreto V 2006 Phys. Rev. E 73026103

[17] Stauffer D and Sahimi M 2005 Phys. Rev. E 72046128

[18] Zlatanov N and Kocarev L 2009 Phys. Rev. E 80041102

[19] Tejedor V, Bénichou O and Voituriez R 2009 Phys. Rev. E 80 065104(R)

[20] Baronchelli A and Pastor-Satorras R 2010 Phys. Rev. E 82 011111

[21] Noh J D, Shim G M and Lee H 2005 Phys. Rev. Lett. 94198701

[22] Noh J D 2005 Phys. Rev. E 72056123

[23] Tang M, Liu Z and Zhou J 2006 Phys. Rev. E 74036101

Tang M and Liu Z 2008 Physica A 3871361

Tang M and Liu Z 2008 Commun. Theo. Phys. 49252

Tang M, Liu Z, Zhu X and Wu X 2008 Int. J. Mod. Phys. C 19 927

[24] Waclaw B, Bogacz L, Burda Z and Janke W 2007 Phys. Rev. E 76046114

[25] Tang M, Liu L and Liu Z 2009 Phys. Rev. E 79016108

[26] Burda Z, Johnston D, Jurkiewicz J, Kaminski M, Nowak M A, Papp G and Zahed I 2002 Phys. Rev. E 65026102 
[27] Evans M R 1996 Europhys. Lett. 3613

[28] Chowdhury D, Santen L and Schadschneider A 2000 Phys. Rep. 329199

[29] Zhu X, Liu Z and Tang M 2007 Chin. Phys. Lett. 242142

[30] Shen J 2010 Chin. Phys. Lett. 27028901

[31] Eggers J 1999 Phys. Rev. Lett. 835322

[32] van der Meer D, van der Meer K and Lohse D 2004 J Stat MechTheory E 404004

[33] Krapivsky P L, Redner S and Leyvraz F 2000 Phys. Rev. Lett. 854629
[34] Bianconi G and Barabási A L 2001 Phys. Rev. Lett. 865632

[35] Tang M, Liu L and Li B 2010 CHAOS 20043135

[36] Bouchaud J P 1992 J. Phys. I 21705 Monthus C and Bouchaud J P 1996 J. Phys. A 293847

[37] Baronchelli A, Barrat A and Pastor-Satorras R 2009 Phys. Rev. E 80 020102(R)

[38] Catanzaro M, Boguñá M and Pastor-Satorras R 2005 Phys. Rev. E 71027103

[39] Cohen R and Havlin S 2003 Phys. Rev. Lett. 90058701 